\title{
Impact of Race and Socioeconomic Status on Outcomes in Patients Hospitalized with COVID-19
}

\author{
Daniel Quan, $B S^{7}$, Lucía Luna Wong, $P h D^{7}$, Anita Shallal, $M D^{2}$, Raghav Madan, BS , \\ Abel Hamdan, BS ', Heaveen Ahdi, BS ', Amir Daneshvar, BS ${ }^{l}$, Manasi Mahajan, BS', \\ Mohamed Nasereldin, $M S^{7}$, Meredith Van Harn, $M S^{3}$, ljeoma Nnodim Opara, $\mathrm{MD}^{4}$, and \\ Marcus Zervos, MD ${ }^{5,6}$
}

\begin{abstract}
'Wayne State University School of Medicine, Detroit, MI, USA; ${ }^{2}$ Department of Infectious Disease, Henry Ford Hospital, Detroit, MI, USA; ${ }^{3}$ Department of Public Health Sciences, Henry Ford Hospital, Detroit, MI, USA; ${ }^{4}$ Department of Internal Medicine, Internal Medicine-Pediatrics Section, Wayne State University School of Medicine, Detroit, MI, USA; ${ }^{5} \mathrm{Global}$ Affairs Professor of Medicine, Assistant Dean Wayne State University School of Medicine, , MIDetroit, USA; IInfectious Diseases, Division Head Henry Ford Health System, , MIDetroit, USA.
\end{abstract}

BACKGROUND The impact of race and socioeconomic status on clinical outcomes has not been quantified in patients hospitalized with coronavirus disease 2019 (COVID-19).

OBJECTIVE To evaluate the association between patient sociodemographics and neighborhood disadvantage with frequencies of death, invasive mechanical ventilation (IMV), and intensive care unit (ICU) admission in patients hospitalized with COVID- 19.

DESIGN Retrospective cohort study.

SETTING Four hospitals in an integrated health system serving southeast Michigan.

PARTICIPANTS Adult patients admitted to the hospital with a COVID-19 diagnosis confirmed by polymerase chain reaction.

MAIN MEASURES Patient sociodemographics, comorbidities, and clinical outcomes were collected. Neighborhood socioeconomic variables were obtained at the census tract level from the 2018 American Community Survey. Relationships between neighborhood median income and clinical outcomes were evaluated using multivariate logistic regression models, controlling for patient age, sex, race, Charlson Comorbidity Index, obesity, smoking status, and living environment.

KEY RESULTS Black patients lived in significantly poorer neighborhoods than White patients (median income: $\$ 34,758(24,531-56,095)$ vs. \$63,317 (49,850-85,776), $p<0.001)$ and were more likely to have Medicaid insurance $(19.4 \%$ vs. $11.2 \%, p<0.001)$. Patients from neighborhoods with lower median income were significantly more likely to require IMV (lowest quartile: 25.4\%, highest quartile: $16.0 \%, p<0.001)$ and ICU admission (35.2\%, $19.9 \%, p<0.001)$. After adjusting for age, sex, race, and comorbidities, higher neighborhood income $\mathbf{\$} \mathbf{\$ 1 0 , 0 0 0}$ increase) remained a significant negative predictor for IMV (OR: 0.95 (95\% CI 0.91, 0.99), $p=0.02$ ) and ICU admission (OR: 0.92 (95\% CI 0.89, 0.96), $p<0.001)$.

Prior Presentations A portion of this work was presented as a Late Breaker Oral Presentation at ID WEEK 2020, and as 2 poster presentations at the American College of Physicians Michigan Chapter Virtual Meetina.

Received August 16, 2020

Accepted December 20, 2020

Published online January 27, 2021
CONCLUSIONS Neighborhood disadvantage, which is closely associated with race, is a predictor of poor clinical outcomes in COVID-19. Measures of neighborhood disadvantage should be used to inform policies that aim to reduce COVID-19 disparities in the Black community.

KEY WORDS COVID-19; disparities; disadvantage; socioeconomic status; race.

J Gen Intern Med 36(5):1302-9

DOI: $10.1007 / \mathrm{s} 11606-020-06527-1$

(C) The Author(s) 2021

\section{INTRODUCTION}

As of October 23, 2020, there are more than 8.3 million confirmed cases and 221,000 deaths from coronavirus disease 2019 (COVID-19) in the USA. ${ }^{1}$ Non-White Americans, especially Black Americans, have been disproportionately affected by the pandemic. In the state of Michigan, Black Americans represent $37 \%$ of COVID-19 cases and $42 \%$ of deaths, despite making up $14 \%$ of Michigan's population. ${ }^{2-4}$ These discrepancies have been largely attributed to social and health disparities rendering Black Americans particularly vulnerable to this novel coronavirus. This explanation is supported by reports that most patients who have been hospitalized and died from COVID-19 have medical comorbidities (e.g., hypertension, diabetes, chronic obstructive pulmonary disease (COPD), heart disease, obesity) that are disproportionately prevalent in the Black community. ${ }^{4-8}$

The effects of medical comorbidities, age, and sex on prognosis in COVID-19 have been widely examined and replicated in previous studies. ${ }^{5,7-10}$ However, systematic examination of race and socioeconomic underpinnings of the COVID-19 pandemic has been lacking. One study showed an increase in the likelihood of hospital admission for COVID-19 in Black individuals and those who lived in low-income areas. ${ }^{7}$ Socioeconomic disadvantage is multifaceted, encompassing economic, educational, social, housing, and healthcare disparities. When combined with patient-level risk factors, 
neighborhood socioeconomic variables have been used to increase the accuracy of predictive models, including risk of preterm birth, ${ }^{11}$ depression, ${ }^{12}$ and cardiovascular disease mortality. ${ }^{13}$

Research into how social determinants can shape COVID19 outcomes is needed. Our study seeks to fill this gap by examining race and patient-level socioeconomic variables, including social support, living environment, and employment status. The objective was to determine whether race and socioeconomic status were associated with clinical outcomes in patients hospitalized with COVID-19. We analyzed the association between patient sociodemographics and census tract socioeconomic variables and frequencies of death, invasive mechanical ventilation (IMV), and intensive care unit (ICU) admission in hospitalized patients. Our aim is to contribute new data that might help inform policies to reduce disparities in the health system.

\section{METHODS}

\section{Study Design and Participants}

This is a retrospective cohort study approved by the Henry Ford Health System Institutional Review Board (IRB \#13843). The Henry Ford Health System is a large, integrated healthcare system serving southeast Michigan. Eligible patients were adult patients admitted to four large hospitals within the Henry Ford Health System from March 12, 2020, to April 24, 2020, inclusive of these dates, and had a positive SARS-CoV-2 test by qualitative polymerase chain reaction. This time period encompasses the rise and peak of the first surge of the pandemic in southeast Michigan. For patients with multiple admissions within the study period, data from the longest admission was presented due to a higher probability of experiencing the clinical outcomes of interest. Patients were followed until May 27, 2020, inclusive. Follow-up encounters consisted of phone calls, video, and in-person visits which verified patients' survival status.

\section{Data Collection}

Clinical data were extracted from the Henry Ford electronic medical record through patient chart review. Our data extraction included the following: patient demographics (age, sex, race, ethnicity, English language proficiency, insurance status, and living situation), employment status for patients $\leq 65$ years, smoking status, substance use, body mass index (BMI), comorbidities, and clinical outcomes. Essential industries included healthcare, agriculture, food service, first responders, transportation, infrastructure, and critical manufacturing. ${ }^{14}$ Medical comorbidities and the Charlson Comorbidity Index (CCI) were obtained by examining the medical history, initial history, and outside care records.

Census tracts from patient addresses were used to obtain socioeconomic data from the 2018 American Community
Survey and the Food Access Research Atlas, ${ }^{15,16}$ data which has been validated and used in health disparities research. ${ }^{17-19}$ The poverty rate obtained from the Food Access Research Atlas is defined as the share of the tract population with income at or below federal poverty thresholds by family size. Census tract median income was used as a numerical surrogate for neighborhood socioeconomic status because of its ease of interpretation and significant correlation with other neighborhood socioeconomic variables.

Presenting symptoms, vital signs, initial laboratory values, and hospital course data were also collected and provided in Appendix Tables 2-5.

\section{Outcomes}

The primary outcomes were death, invasive mechanical ventilation, and ICU admission anytime during hospitalization.

\section{Statistical Analysis}

Continuous variables were described as mean (SD) or median (Q1-Q3). Categorical variables were described with counts and percentages. Univariate comparisons between groups were carried out using independent two-group $t$ tests for normally distributed continuous variables and using Wilcoxon rank-sum tests for non-normally distributed continuous variables. Categorical variables were compared using chi-square or Fisher's exact test based on expected cell count. Multivariable logistic regression models were used to identify possible independent predictors of death, ventilator use, and ICU admission with results presented as adjusted odds ratios and 95\% confidence intervals. Increased age, male sex, Black race, increased comorbidity burden, obesity, and smoking were incorporated into the multivariable model because they have been associated with death and severe disease in previous studies. ${ }^{7-10}$ When post hoc pairwise testing was performed, the type I error rate was controlled using a BenjaminiHochberg correction and adjusted $p$ values were reported. All analyses were performed using SAS 9.4 (SAS Institute Inc, Cary, NC, USA).

\section{RESULTS}

\section{Patient Demographics}

Of the 2038 patients hospitalized, 1209 (59.3\%) were Black and $694(34.1 \%)$ were White. The mean age of the hospitalized patients was 64 years, and approximately half were female (Table 1). Black patients comprised a larger proportion of each hospital's patients than the surrounding city's Black population. The proportion of Black patients was $90.2 \%$ in Henry Ford Hospital, 35.5\% in Henry Ford Macomb, 53.0\% in Henry Ford West Bloomfield, and 39.0\% in Henry Ford Wyandotte. Conversely, census data shows the Black population comprises $77 \%$ of the population in Detroit, $18 \%$ in Macomb, $13 \%$ in West Bloomfield, and $1 \%$ in Wyandotte. ${ }^{20}$ 
Table 1 Socioeconomic and Environmental Characteristics of Alive and Dead Patients Hospitalized with COVID-19

\begin{tabular}{|c|c|c|c|}
\hline Characteristic & $\begin{array}{l}\text { All } \\
(n=2038)\end{array}$ & $\begin{array}{l}\text { Alive } \\
(n=1587)\end{array}$ & $\begin{array}{l}\text { Dead } \\
(n=442)\end{array}$ \\
\hline Age, mean (SD), years & $\begin{array}{l}63.96 \\
(16.23)\end{array}$ & $60.9(15.6)$ & $75.1(13.6)$ \\
\hline Female, no. $(\%)$ & $1027(50.4)$ & $824(51.95)$ & $201(45.48)$ \\
\hline $\begin{array}{l}\text { Length of stay, days } \\
\text { (Q1-Q3) }\end{array}$ & $6(3-12)$ & $6(3-10)$ & $9(4-15)$ \\
\hline $\begin{array}{l}\text { Readmission, no. (\%) } \\
\text { Race, no. }(\%)\end{array}$ & & $54(12.22)$ \\
\hline White & $694(34.1)$ & $491(30.94)$ & $201(45.48)$ \\
\hline Black & $1209(59.3)$ & $984(62)$ & $218(49.32)$ \\
\hline Asian/Pacific Islander & $39(1.9)$ & $32(2.02)$ & $7(1.58)$ \\
\hline Native American & $2(0.1)$ & $1(0.06)$ & $1(0.23)$ \\
\hline Other/missing & $94(4.6)$ & $79(4.98)$ & $15(3.39)$ \\
\hline \multicolumn{4}{|l|}{ Living situation, no. (\%) } \\
\hline With family & $1210(66.2)$ & $969(69.86)$ & $234(54.29)$ \\
\hline Alone & & 217( & $49(11.37)$ \\
\hline Group facility & 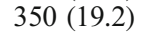 & $201(14.49)$ & $148(34.34)$ \\
\hline \multicolumn{4}{|c|}{ Insurance status, no. (\%) } \\
\hline Commercial insurance & $494(26.9)$ & $461(32.24)$ & $32(8.04)$ \\
\hline Medi & $958(52.2)$ & $629(43.99)$ & $324(81.41)$ \\
\hline Medic & 304 (16.6) & $268(18.74)$ & $35(8.79)$ \\
\hline Self- 1 & $4(0.2)$ & $4(0.28)$ & $0(0)$ \\
\hline Other & $21(1.1)$ & $19(1.33)$ & $2(0.5)$ \\
\hline No ins & $54(2.9)$ & $49(3.43)$ & $5(1.26)$ \\
\hline \multicolumn{4}{|c|}{ Medical conditions, no. (\%) } \\
\hline BMI, mean (SD), $\mathrm{kg} / \mathrm{m}^{2}$ & $31.75(8.66)$ & $32.41(8.53)$ & $29.3(8.61)$ \\
\hline & \multicolumn{2}{|c|}{ Index, mean (SD) } & $3.57(2.54)$ \\
\hline Hypertension & $1494(73.3)$ & $\begin{array}{l}1121 \\
(70.64)\end{array}$ & $364(82.54)$ \\
\hline Psyc & 520 & $384(24$ & 134 \\
\hline $\begin{array}{l}\text { Coronary artery } \\
\text { disease }\end{array}$ & 308 & $197(12.41)$ & $108(24.49)$ \\
\hline $\begin{array}{l}\text { Congestive heart } \\
\text { failure }\end{array}$ & $276(13.5)$ & $162(10.21)$ & $(25.17)$ \\
\hline $\begin{array}{l}\text { Cerebrovascular } \\
\text { disease }\end{array}$ & $271(13.3)$ & $163(10.27)$ & $107(24.26)$ \\
\hline COPD & 294 & 176 & 115 \\
\hline Diabetes & 652 & 490 & $157(35.6)$ \\
\hline $\begin{array}{l}\text { Diabetes with } \\
\text { complications }\end{array}$ & $132(6.5)$ & $90(5.67)$ & $40(9.07)$ \\
\hline $\begin{array}{l}\text { Chronic kidney } \\
\text { disease }\end{array}$ & $357(17.5)$ & $216(13.61)$ & $136(30.84)$ \\
\hline Malignancy & 136 & 88 & $48(10.88)$ \\
\hline cancer & $26(1.3)$ & 10( & $16(3.63)$ \\
\hline Tobacco use & $810(43.1)$ & $589(39.96)$ & $218(54.64)$ \\
\hline Substance use & $186(10.3)$ & $146(10.3)$ & $39(10)$ \\
\hline \multicolumn{4}{|c|}{ Neighborhood characteristics, \% (SD) } \\
\hline $\begin{array}{l}\text { Bachelor's degree or } \\
\text { higher }\end{array}$ & $18(13)$ & $18(13)$ & $18(13)$ \\
\hline Less than high school & 9( & 9 & $9(5)$ \\
\hline Median income & 47,645 & 47,363 & 49,850 \\
\hline (Q1-Q3) & $\begin{array}{l}(29,541- \\
68,924)\end{array}$ & $\begin{array}{l}(29,721- \\
70,104)\end{array}$ & $\begin{array}{l}(29,721- \\
148,875)\end{array}$ \\
\hline Rent & $37(22)$ & $37(22)$ & $37(21)$ \\
\hline Unemployment & $5(3)$ & $5(3)$ & $5(4)$ \\
\hline Poverty $\mathrm{r}$ & $24(18)$ & $24(18)$ & $23(17)$ \\
\hline SNAP recipien & $24(17)$ & $24(18)$ & $23(17)$ \\
\hline No vehicle access & $14(13)$ & $14(14)$ & $14(12)$ \\
\hline
\end{tabular}

Q1-Q3, first quartile-third quartile; SD, standard deviation; BMI, body mass index; COPD, chronic obstructive pulmonary disease; SNAP, Supplemental Nutrition Assistance Program

\section{Patient Outcomes}

At follow-up, 1587 patients were discharged alive, 442 had died, and 9 were still admitted. A total of 405 patients died in the hospital (91.6\%), of which 182 were White and 202 were Black. The median follow-up time for patients discharged alive was 27 days (3-37) and median time to death for patients who died outside the hospital was 12 days (6-23). The median duration of hospitalization for the nine patients still admitted was 46 days (46-49). The case fatality rate (CFR) for all patients was $21.7 \%$ and White patients had a higher CFR than Black patients $(29.1 \%$ vs. $18.1 \%, p<0.001)$ (Table 2$)$. Conversely, Black patients were more likely than White patients to receive mechanical ventilation $(23.6 \%$ vs. $19.7 \%, p=0.053)$ and require ICU level care (31.4\% vs. $25.5 \%, p=0.003)$. Of the 455 patients who received mechanical ventilation, 215 (47.2\%) were discharged alive, 231 (50.8\%) died, and 9 were still admitted.

Patients who died were significantly older than those discharged alive $(75.1 \pm 13.6$ years vs. $60.9 \pm 15.6$ years, $p<$ 0.001 ) and had more comorbidities (CCI: $3.6 \pm 2.5$ vs. $1.8 \pm$

Table 2 Socioeconomic and Environmental Characteristics of Black and White Patients

\begin{tabular}{|c|c|c|}
\hline Characteristic & $\begin{array}{l}\text { White } \\
(n=694)\end{array}$ & $\begin{array}{l}\text { Black } \\
(n=1209)\end{array}$ \\
\hline Age, mean (SD), years & $68.6(16.7)$ & $61.7(15.5)$ \\
\hline Female, no. $(\%)$ & $341(49.14)$ & $635(52.57)$ \\
\hline \multicolumn{3}{|l|}{ Clinical outcomes, no. (\%) } \\
\hline Death & $201(29.05)$ & $218(18.14)$ \\
\hline Mechanical ventilation & $137(19.74)$ & $285(23.57)$ \\
\hline ICU admission & $174(25.07)$ & $380(31.43)$ \\
\hline Length of stay, days & $6(4-12)$ & $6(3-12)$ \\
\hline $\begin{array}{l}\text { (Q1-Q3) } \\
\text { Readmission, no. (\%) }\end{array}$ & $107(15.42)$ & $114(9.43)$ \\
\hline \multicolumn{3}{|l|}{ Living situation, no. (\%) } \\
\hline With family & $381(59.25)$ & $744(69.6)$ \\
\hline Alone & $65(10.11)$ & $184(17.21)$ \\
\hline \multirow{2}{*}{\multicolumn{3}{|c|}{ Insurance status, no. (\%) }} \\
\hline & & \\
\hline Commercial insurance & $151(23.74)$ & 305 (27.9) \\
\hline Medicare & $392(61.64)$ & $533(48.76)$ \\
\hline Medicaid & $71(11.16)$ & $212(19.4)$ \\
\hline Self-pay & $1(0.16)$ & $2(0.18)$ \\
\hline Other & $7(1.1)$ & $12(1.1)$ \\
\hline No insurance & $14(2.2)$ & $29(2.65)$ \\
\hline \multicolumn{3}{|l|}{ Medical conditions, no. (\%) } \\
\hline BMI, mean (SD), $\mathrm{kg} / \mathrm{m}^{2}$ & $29.9(7.8)$ & $33.0(8.9)$ \\
\hline $\begin{array}{l}\text { Charlson Comorbidity Index, } \\
\text { mean (SD) }\end{array}$ & $2.35(2.18)$ & $2.22(2.18)$ \\
\hline Hypertension & $474(68.4)$ & $932(77.09)$ \\
\hline Psychiatric condition & $232(33.48)$ & $268(22.17)$ \\
\hline Coronary artery disease & $151(21.79)$ & $146(12.08)$ \\
\hline Congestive heart failure & $104(15.01)$ & $162(13.4)$ \\
\hline Cerebrovascular disease & $101(14.57)$ & 158 (13.07) \\
\hline COPD & 119 (17.17) & $165(13.65)$ \\
\hline Diabetes & $185(26.7)$ & $413(34.16)$ \\
\hline Diabetes with complications & $37(5.34)$ & $92(7.61)$ \\
\hline Chronic kidney disease & $112(16.16)$ & $235(19.44)$ \\
\hline Malignancy & $52(7.5)$ & $73(6.04)$ \\
\hline Metastatic cancer & $10(1.44)$ & $14(1.16)$ \\
\hline Tobacco use & $322(49.92)$ & $463(41.34)$ \\
\hline Substance use & $47(7.62)$ & $131(12.01)$ \\
\hline \multicolumn{3}{|c|}{ Neighborhood characteristics, \% (SD) } \\
\hline Bachelors or higher & $22(13)$ & $15(13)$ \\
\hline Less than high school & $7(5)$ & $10(6)$ \\
\hline Median income (Q1-Q3) & $\begin{array}{l}63,317(49,850- \\
85,776)\end{array}$ & $\begin{array}{l}34,758(24,531- \\
56,095)\end{array}$ \\
\hline Renters & 26 (19) & $44(21)$ \\
\hline Unemployment & $3(2)$ & $6(3)$ \\
\hline Poverty rate & $13(11)$ & $30(18)$ \\
\hline SNAP recipients & $13(12)$ & $30(17)$ \\
\hline No vehicle access & $7(8)$ & $19(14)$ \\
\hline
\end{tabular}

Q1-Q3, first quartile-third quartile; SD, standard deviation; ICU, intensive care unit; BMI, body mass index; $C O P D$, chronic obstructive pulmonary disease; SNAP, Supplemental Nutrition Assistance Program 
$1.9, p<0.001)$ (Table 1). They had higher rates of hypertension, psychiatric conditions, cardiovascular disease, COPD, chronic kidney disease (CKD), and cancer. White patients were significantly older than Black patients $(68.6 \pm 16.7$ years vs. $61.7 \pm 15.5$ years, $p<0.001$ ), although the CCI was similar among the two races $(2.4 \pm 2.2$ vs. $2.2 \pm 2.2, p=0.21)$ (Table 4). White patients were more likely to have a history of coronary artery disease and psychiatric conditions. Black patients were more likely to have a history of hypertension, diabetes, and obesity. Patients who died had lower BMI, but those who received IMV and ICU care had higher BMI (Tables 1, 2, and 3). Presenting symptoms, vital signs, initial laboratory values, and hospital course data are provided in the Appendix. Patients with commercial insurance had lower rates

Table 4 Socioeconomic and Environmental Characteristics of Patients Requiring ICU Admission

\begin{tabular}{|c|c|c|}
\hline Characteristic & $\begin{array}{l}\text { No ICU } \\
(n=1452)\end{array}$ & $\begin{array}{l}\mathrm{ICU} \\
(n=586)\end{array}$ \\
\hline Age, mean (SD), years & $63.5(17.0)$ & $65.1(14.2)$ \\
\hline Female, no. $(\%)$ & $767(52.86)$ & $260(44.37)$ \\
\hline Length of stay, days (Q1-Q3) & $5(3-8)$ & $17(23-62)$ \\
\hline \multirow{2}{*}{\multicolumn{3}{|c|}{ Race, no. $(\%)$}} \\
\hline & & \\
\hline White & $520(35.81)$ & $174(29.69)$ \\
\hline Black & $829(57.09)$ & $380(64.85)$ \\
\hline Asian/Pacific Islander & $26(1.79)$ & $13(2.22)$ \\
\hline Native American & $2(0.14)$ & $0(0)$ \\
\hline Other/missing & $75(5.17)$ & $19(3.24)$ \\
\hline \multicolumn{3}{|l|}{ Living situation, no. (\%) } \\
\hline With family & $801(63.93)$ & 409 (71.25) \\
\hline Alone & $190(15.16)$ & $77(13.41)$ \\
\hline Group facility & $262(20.91)$ & $88(15.33)$ \\
\hline \multicolumn{3}{|l|}{ Insurance status, no. (\%) } \\
\hline Commercial insurance & $392(30.2)$ & $102(18.99)$ \\
\hline Medicare & $633(48.77)$ & $325(60.52)$ \\
\hline Medicaid & $213(16.41)$ & $91(16.95)$ \\
\hline Self-pay & $4(0.31)$ & $0(0)$ \\
\hline Other & $17(1.31)$ & $4(0.74)$ \\
\hline No insurance & $39(3)$ & $15(2.79)$ \\
\hline \multicolumn{3}{|l|}{ Medical conditions, no. (\%) } \\
\hline BMI, mean (SD), $\mathrm{kg} / \mathrm{m}^{2}$ & $31.2(8.2)$ & $33.1(9.5)$ \\
\hline $\begin{array}{l}\text { Charlson Comorbidity Index, } \\
\text { mean (SD) }\end{array}$ & $1.99(2.05)$ & $2.80(2.39)$ \\
\hline Hypertension & $1019(70.23)$ & $475(81.06)$ \\
\hline Psychiatric condition & $365(25.16)$ & $155(26.45)$ \\
\hline Coronary artery disease & $191(13.16)$ & $117(19.97)$ \\
\hline Congestive heart failure & $173(11.92)$ & $103(17.58)$ \\
\hline Cerebrovascular disease & $188(12.96)$ & $83(14.16)$ \\
\hline COPD & 169 (11.65) & $125(21.33)$ \\
\hline Diabetes & $423(29.15)$ & $229(39.08)$ \\
\hline Diabetes with complications & $74(5.1)$ & $58(9.9)$ \\
\hline Chronic kidney disease & $220(15.16)$ & $137(23.38)$ \\
\hline Malignancy & $88(6.06)$ & $48(8.19)$ \\
\hline Metastatic cancer & $14(0.96)$ & $12(2.05)$ \\
\hline Tobacco use & $549(40.7)$ & $261(49.15)$ \\
\hline Substance use & $134(10.35)$ & $52(10.02)$ \\
\hline \multicolumn{3}{|c|}{ Neighborhood characteristics, \% (SD) } \\
\hline Bachelor's degree or higher & 19 (14) & $15(12)$ \\
\hline Less than high school & $9(6)$ & $10(6)$ \\
\hline Median income (Q1-Q3) & $\begin{array}{l}49,867(30,417- \\
72,868)\end{array}$ & $\begin{array}{l}38,060(26,602- \\
61,111)\end{array}$ \\
\hline Renters & $36(22)$ & $40(21)$ \\
\hline Unemployment & $4(3)$ & $5(4)$ \\
\hline Poverty rate & $22(18)$ & $27(18)$ \\
\hline SNAP recipients & $23(17)$ & $27(17)$ \\
\hline No vehicle access & $14(13)$ & 17 (13) \\
\hline
\end{tabular}

Q1-Q3, first quartile-third quartile; SD, standard deviation; ICU, intensive care unit; BMI, body mass index; $C O P D$, chronic obstructive pulmonary disease; SNAP, Supplemental Nutrition Assistance Program
Table 3 Socioeconomic and Environmental Characteristics of Patients Requiring Mechanical Ventilation

\begin{tabular}{|c|c|c|}
\hline Characteristic & $\begin{array}{l}\text { No IMV } \\
(n=1596)\end{array}$ & $\begin{array}{l}\text { IMV } \\
(n=442)\end{array}$ \\
\hline Age, mean (SD), years & $63.5(16.8)$ & $65.5(14.0)$ \\
\hline Female, no. (\%) & $836(52.41)$ & $191(43.21)$ \\
\hline Length of stay, days (Q1-Q3) & $6(3-8)$ & $19(10-26)$ \\
\hline Readmission, no. (\%) & $185(11.59)$ & $41(9.28)$ \\
\hline \multicolumn{3}{|l|}{ Race, no. $(\%)$} \\
\hline White & $557(34.9)$ & $137(31)$ \\
\hline Black & $924(57.89)$ & $285(64.48)$ \\
\hline Asian/Pacific Islander & $29(1.82)$ & $10(2.26)$ \\
\hline Native American & $2(0.13)$ & $0(0)$ \\
\hline Other/missing & $84(5.26)$ & $10(2.26)$ \\
\hline \multicolumn{3}{|l|}{ Living situation, no. (\%) } \\
\hline With family & $902(64.75)$ & $308(70.97)$ \\
\hline Alone & $207(14.86)$ & $60(13.82)$ \\
\hline Group facility & $284(20.39)$ & $66(15.21)$ \\
\hline \multicolumn{3}{|l|}{ Insurance status, no. (\%) } \\
\hline Commercial insurance & $417(29.2)$ & $77(18.92)$ \\
\hline Medicare & 709 (49.65) & $249(61.18)$ \\
\hline Medicaid & $238(16.67)$ & $66(16.22)$ \\
\hline Self-pay & $4(0.28)$ & $0(0)$ \\
\hline Other & $19(1.33)$ & $2(0.49)$ \\
\hline No insurance & $41(2.87)$ & $13(3.19)$ \\
\hline \multicolumn{3}{|l|}{ Medical conditions, no. (\%) } \\
\hline BMI, mean (SD), $\mathrm{kg} / \mathrm{m}^{2}$ & $31.3(8.3)$ & $33.3(9.8)$ \\
\hline $\begin{array}{l}\text { Charlson Comorbidity Index, } \\
\text { mean (SD) }\end{array}$ & $2.06(2.10)$ & $2.81(2.36)$ \\
\hline Hypertension & $1131(70.91)$ & $363(82.13)$ \\
\hline Psychiatric condition & $402(25.2)$ & $118(26.7)$ \\
\hline Coronary artery disease & $218(13.67)$ & $90(20.36)$ \\
\hline Congestive heart failure & $193(12.1)$ & $83(18.78)$ \\
\hline Cerebrovascular disease & $207(12.98)$ & $64(14.48)$ \\
\hline COPD & 199 (12.48) & $95(21.49)$ \\
\hline Diabetes & $471(29.53)$ & $181(40.95)$ \\
\hline Diabetes with complications & $89(5.58)$ & $43(9.73)$ \\
\hline Chronic kidney disease & $253(15.86)$ & $104(23.53)$ \\
\hline Malignancy & $97(6.08)$ & $39(8.82)$ \\
\hline Metastatic cancer & $17(1.07)$ & $9(2.04)$ \\
\hline Tobacco use & $609(41.07)$ & $201(50.63)$ \\
\hline Substance use & $146(10.27)$ & $40(10.2)$ \\
\hline \multicolumn{3}{|c|}{ Neighborhood characteristics, \% (SD) } \\
\hline Bachelor's degree or higher & $19(14)$ & $16(12)$ \\
\hline Less than high school & $9(6)$ & $10(6)$ \\
\hline Median income (Q1-Q3) & $\begin{array}{l}48,680(30,050- \\
72,434)\end{array}$ & $\begin{array}{l}40,488(27,679 \\
62,992)\end{array}$ \\
\hline Renters & $37(22)$ & $39(21)$ \\
\hline Unemployment & $5(3)$ & $5(4)$ \\
\hline Poverty rate & $23(18)$ & $26(18)$ \\
\hline SNAP recipients & $23(18)$ & $26(17)$ \\
\hline No vehicle access & $14(13)$ & $16(13)$ \\
\hline
\end{tabular}

Q1-Q3, first quartile-third quartile; SD, standard deviation; IMV, invasive mechanical ventilation; BMI, body mass index; $C O P D$, chronic obstructive pulmonary disease; SNAP, Supplemental Nutrition Assistance Program

of death, IMV, and ICU admission than those with Medicaid insurance. Those who lived in group facilities had a higher risk of death, but lower frequencies of IMV and ICU admission. A larger proportion of White patients lived in group facilities than Black patients $(30.6 \%$ vs. $13.2 \%, p<0.001)$ (Table 4$)$.

\section{Neighborhood Effects}

Patients who died did not live in significantly disadvantaged neighborhoods, with regard to median income, unemployment, and educational attainment (Table 1). However, patients who received IMV and ICU care lived in neighborhoods with significantly lower median income, educational attainment, and vehicle access, and higher unemployment rates and food 
insecurity (Tables 2 and 3). Black patients lived in significantly poorer neighborhoods than White patients (median income: $\$ 34,758(24,531-56,095)$ vs. $\$ 63,317(49,850-85,776), p<$ 0.001 ) (Table 4). The neighborhoods that Black patients lived in also reported lower educational attainment (bachelor's degree or higher: $15.2 \%$ vs. $21.9 \%, p<0.001$ ), higher percentage of renters ( $43.9 \%$ vs. $25.9 \%, p<0.001)$, higher unemployment rate $(5.8 \%$ vs. $2.8 \%, p<0.001)$, higher poverty rate $(29.2 \%$ vs. $12.6 \%, p<0.001)$, more Supplemental Nutrition Assistance Program (SNAP) benefits recipients (30.4\% vs. $13.1 \%, p<$ $0.001)$, and more households without vehicle access $(18.6 \%$ vs. $7.4 \%, p<0.001)$. Black patients were almost twice as likely as White patients to have Medicaid insurance (19.4\% vs. $11.2 \%, p<0.001)$.

Analysis of outcomes by median income quartiles showed that patients from poorer neighborhoods had significantly higher frequencies of mechanical ventilation and ICU admission compared to patients from wealthier neighborhoods (Table 5). Stratification by Black or White race and median income quartiles revealed that among Black patients, those who lived in poorer neighborhoods had higher frequencies of death, mechanical ventilation, and ICU admission compared to Black patients living in wealthier neighborhoods. Among White patients, those living in disadvantaged neighborhoods had higher rates of ICU admission but similar rates of death and mechanical ventilation, when compared to White patients living in wealthier neighborhoods.

\section{Multivariate Analysis of Risk Factors for Mortality and Severe Disease}

Table 6 shows the results of multivariate analyses adjusting for baseline characteristics. We used a model incorporating age (1-year increase), male sex, Black race, CCI (1-unit increase),

Table 5 Clinical Outcomes Stratified by Neighborhood Median Income Quartiles

\begin{tabular}{llllll}
\hline \hline & $\leq$ & $\mathbf{\$ 2 9 . 6 K}-$ & $\mathbf{\$ 4 7 . 6 K}-$ & $\mathbf{Z}$ & $\boldsymbol{p}$ \\
& $\mathbf{\$ 2 9 . 5 K}$ & $\mathbf{4 7 . 6 K}$ & $\mathbf{\$ 6 8 . 9 K}$ & $\mathbf{\$ 6 8 . 9 K}$ & value \\
\hline All patients & & & & & \\
Death, & 110 & 104 & 123 & 105 & 0.489 \\
no. (\%) & $(21.7 \%)$ & $(20.6 \%)$ & $(24.1 \%)$ & $(20.7 \%)$ & \\
IMV, no. & 130 & 126 & 105 & 81 & $<$ \\
(\%) & $(25.4 \%)$ & $(24.8 \%)$ & $(20.5 \%)$ & $(16.0 \%)$ & 0.001 \\
ICU, no. & 180 & 170 & 135 & 101 & $<$ \\
$(\%)$ & $(35.2 \%)$ & $(33.5 \%)$ & $(26.4 \%)$ & $(19.9 \%)$ & 0.001 \\
White only & & & & & \\
Death, & 13 & 26 & 88 & 74 & 0.276 \\
no. (\%) & $(34.2 \%)$ & $(25.2 \%)$ & $(32.6 \%)$ & $(26.3 \%)$ & \\
IMV, no. & 11 & 24 & 54 & 48 & 0.259 \\
$(\%)$ & $(29.0 \%)$ & $(23.1 \%)$ & $(19.9 \%)$ & $(17.1 \%)$ & \\
ICU, no. & 16 & 29 & 68 & 61 & 0.046 \\
$(\%)$ & $(42.1 \%)$ & $(27.9 \%)$ & $(25.1 \%)$ & $(21.7 \%)$ & \\
Black only & & & & & \\
Death, & 92 & 74 & 30 & 22 & 0.017 \\
no. (\%) & $(20.8 \%)$ & $(20.4 \%)$ & $(13.8 \%)$ & $(12.3 \%)$ & \\
IMV, no. & 116 & 96 & 48 & 25 & 0.006 \\
$(\%)$ & $(25.9 \%)$ & $(26.4 \%)$ & $(22.0 \%)$ & $(14.0 \%)$ & \\
ICU, no. & 159 & 132 & 61 & 28 & $<$ \\
$(\%)$ & $(35.5 \%)$ & $(36.3 \%)$ & $(28.0 \%)$ & $(15.6 \%)$ & 0.001 \\
\hline
\end{tabular}

$I M V$, invasive mechanical ventilation; ICU, intensive care unit obesity (BMI > $35 \mathrm{~kg} / \mathrm{m}^{2}$ ), smoking status, neighborhood median income ( $\$ 10,000$ increase), and living in a group facility. Increased age, male sex, higher CCI, and living in a group facility were predictors of death. Black race was not a significant predictor of death, even though Black patients had a lower rate of death than White patients.

Male sex, increasing CCI, obesity, and lower neighborhood median income were significant predictors for IMV and ICU admission, while living in a group facility was protective against IMV and ICU admission. Age was not an independent predictor of IMV or ICU. Black race was not an independent predictor of IMV or ICU, even though Black patients had higher rates of mechanical ventilation and ICU admission.

To determine race-specific predictors, we stratified our model by Black and White race (Table 6). Interestingly, male sex was an independent predictor for death, IMV, and ICU admission in Black patients, but not in White patients. Neighborhood median income was a significant predictor for death, IMV and ICU admission in Black patients, but only ICU admission in White patients. Living in a group facility was a predictor for death in White patients, but not Black patients.

\section{DISCUSSION}

The COVID-19 pandemic revealed the health disparities that exist between the Black and White populations of southeast Michigan, a population which can be generalized to other metropolitan areas. This study aims to shed light on the characteristics of these disparities by correlating outcomes with race and socioeconomic factors. An earlier case series conducted by our colleagues described the clinical characteristics and outcomes for the first wave of COVID-19 patients in metropolitan Detroit. ${ }^{8}$ Our study builds on their work while factoring in the socioeconomic variables of a large cohort hospitalized with COVID-19 during the peak of the pandemic in Michigan. Consistent with our results, they conclude that most of the infected hospitalized patients from the first wave of the pandemic were Black. In our study, during the peak of the pandemic, Black patients comprised nearly $60 \%$ of our cohort, despite making up only $14 \%$ of the state's population. This overrepresentation is supported by Black patients representing a larger proportion of each hospital's admissions than respective city demographics. In our study, the CFR was higher in White patients than in Black patients $(29.1 \%$ vs. $18.1 \%$ ), which is likely due to the significantly older age of White patients compared to Black patients (68.6 years vs. 61.7 years). The lower CFR for Black patients suggests that the higher death rate of COVID-19 among the Black population per capita in Michigan and nationally may stem from higher rates of infection per capita, which is supported by analyses of infection rates by race. ${ }^{21,22}$

Social distancing and self-isolation are highly effective in reducing transmission but remain a difficulty for many Black Americans due to housing density, frontline employment, and 
Table 6 Multivariable Analysis of Characteristics Associated with Death, Mechanical Ventilation, and ICU Admission

\begin{tabular}{|c|c|c|c|c|c|c|}
\hline \multirow[t]{2}{*}{ Characteristic } & \multicolumn{2}{|l|}{ Death } & \multicolumn{2}{|l|}{ Mechanical ventilation } & \multicolumn{2}{|l|}{ ICU admission } \\
\hline & Adjusted OR (95\% CI) & $p$ value & Adjusted OR (95\% CI) & $p$ value & Adjusted OR (95\% CI) & $p$ value \\
\hline Age (1-year increase) & $1.06(1.04,1.07)$ & $<0.001$ & $1.01(0.99,1.02)$ & 0.162 & $1.00(0.99,1.01)$ & 0.589 \\
\hline Male sex & $1.47(1.14,1.90)$ & 0.003 & $1.63(1.29,2.07)$ & $<0.001$ & $1.55(1.25,1.92)$ & $<0.001$ \\
\hline Black race & $0.86(0.64,1.14)$ & 0.284 & $1.16(0.89,1.51)$ & 0.284 & $1.13(0.88,1.44)$ & 0.342 \\
\hline CCI (1-unit increase) & $1.23(1.16,1.30)$ & $<0.001$ & $1.13(1.07,1.19)$ & $<0.001$ & $1.16(1.10,1.22)$ & $<0.001$ \\
\hline $\mathrm{BMI}>35$ & $0.96(0.69,1.32)$ & 0.78 & $1.64(1.26,2.14)$ & $<0.001$ & $1.51(1.19,1.94)$ & $<0.001$ \\
\hline Income ( $\$ 10,000$ increase) & $0.97(0.92,1.01)$ & 0.14 & $0.95(0.91,0.99)$ & 0.02 & $0.92(0.89,0.96)$ & $<0.001$ \\
\hline Tobacco use & $1.08(0.84,1.40)$ & 0.547 & $1.20(0.94,1.53)$ & 0.145 & $1.13(0.91,1.41)$ & 0.279 \\
\hline Group facility & $1.61(1.19,2.16)$ & 0.002 & $0.63(0.45,0.88)$ & 0.007 & $0.64(0.47,0.87)$ & 0.004 \\
\hline White patients & Death & & Mechanical ventilation & & ICU admission & \\
\hline Variable & Adjusted OR (95\% CI) & $p$ value & Adjusted OR (95\% CI) & $p$ value & Adjusted OR (95\% CI) & $p$ value \\
\hline Age (1-year increase) & $1.04(1.03,1.06)$ & $<0.001$ & $1.00(0.99,1.02)$ & 0.858 & $0.99(0.98,1.01)$ & 0.541 \\
\hline Male sex & $1.24(0.83,1.85)$ & 0.298 & $1.47(0.97,2.22)$ & 0.068 & $1.39(0.95,2.02)$ & 0.092 \\
\hline CCI (1-unit increase) & $1.22(1.11,1.34)$ & $<0.001$ & $1.13(1.03,1.25)$ & 0.012 & $1.14(1.04,1.25)$ & 0.004 \\
\hline $\mathrm{BMI}>35$ & $0.44(0.23,0.82)$ & 0.01 & $1.42(0.86,2.340$ & 0.175 & $1.26(0.79,2.00)$ & 0.339 \\
\hline Income $(\$ 10,000$ increase $)$ & $0.97(0.90,1.04)$ & 0.344 & $0.94(0.87,1.02)$ & 0.131 & $0.93(0.86,0.99)$ & 0.033 \\
\hline Tobacco use & $0.84(0.56,1.25)$ & 0.382 & $1.16(0.76,1.75)$ & 0.5 & $1.35(0.92,1.97)$ & 0.13 \\
\hline Lives in group facility & $1.92(1.26,2.94)$ & 0.003 & $0.72(0.44,1.17)$ & 0.184 & $0.74(0.47,1.16)$ & 0.193 \\
\hline Black patients & Death & & Mechanical ventilation & & ICU admission & \\
\hline Variable & Adjusted OR (95\% CI) & $p$ value & Adjusted OR (95\% CI) & $p$ value & Adjusted OR (95\% CI) & $p$ value \\
\hline Age (1-year increase) & $1.06(1.04,1.07)$ & $<0.001$ & $1.01(0.99,1.02)$ & 0.196 & $1.00(0.99,1.02)$ & 0.421 \\
\hline Male sex & $1.76(1.24,2.51)$ & 0.002 & $1.76(1.30,2.37)$ & $<0.001$ & $1.66(1.26,2.19)$ & $<0.001$ \\
\hline CCI (1-unit increase) & $1.28(1.18,1.38)$ & $<0.001$ & $1.14(1.07,1.22)$ & $<0.001$ & $1.18(1.10,1.26)$ & $<0.001$ \\
\hline $\mathrm{BMI}>35$ & $1.35(0.90,2.02)$ & 0.15 & $1.74(1.26,2.41)$ & $<0.001$ & $1.62(1.20,2.20)$ & 0.002 \\
\hline Income $(\$ 10,000$ increase $)$ & $0.93(0.87,0.99)$ & 0.032 & $0.93(0.88,0.99)$ & 0.023 & $0.89(0.85,0.95)$ & $<0.001$ \\
\hline Tobacco use & $1.33(0.93,1.90)$ & 0.118 & $1.26(0.92,1.72)$ & 0.149 & $1.08(0.81,1.44)$ & 0.603 \\
\hline Lives in group facility & $1.23(0.78,1.94)$ & 0.38 & $0.59(0.36,0.95)$ & 0.031 & $0.61(0.39,0.93)$ & 0.023 \\
\hline
\end{tabular}

CCI, Charlson Comorbidity Index; BMI, body mass index; Income, neighborhood median income

food deserts, all of which are linked to socioeconomic disparities. $^{23-25}$ Frontline employment comprised a plurality of patients under 65 years, though Black and White patients in our cohort had similar employment characteristics. Finally, we found that living in a group facility is an independent predictor for mortality, even after adjusting for age and comorbidities. This is again, likely due to sharing space and little to no opportunity for proper social distancing and isolation, highlighting the need for reform. Our findings highlight the importance of the living and social environment on COVID-19 outcomes and is reflected in the devastation that the pandemic has wrought upon nursing facilities in southeast Michigan and impoverished communities. ${ }^{26,27}$

Multivariable analysis found increased age, male sex, and comorbidities but not race to be significant predictors of mortality, consistent with other reports. ${ }^{5,70}$ Hypertension, cardiovascular disease, COPD, diabetes, CKD, and cancer were associated with death, as was increased CCI. Although CCI for White and Black patients were similar, the comorbidities disproportionately prevalent in the Black populationhypertension, diabetes, and CKD - were associated with a worse prognosis. The higher prevalence of these conditions did not result in higher mortality in hospitalized Black patients, but may account for higher hospital admission rates, consistent with comparative analyses of hospitalized and discharged patients. ${ }^{7,8}$ In the multivariate analysis, Black race was not a significant predictor for IMV or ICU admission, consistent with analyses from an earlier study in our health system. ${ }^{8}$ Obesity was an independent predictor of need for IMV and ICU care, consistent with other reports. ${ }^{28,29}$ Notably, Black patients had significantly higher BMI than White patients (BMI: 33.0 vs. 29.8, $p<0.001$ ). Higher rates of obesity among the Black population in the USA have been linked to higher prevalence of food deserts and food insecurity in communities of color. ${ }^{30-32}$ Finally, the finding that male sex was a more significant predictor in Black patients highlights the unique stresses of the Black male experience in the USA, many of which were not captured in our analysis. These stresses include police violence, mass incarceration, and health and education disparities. ${ }^{33-38}$

When neighborhood income quartiles were stratified by race, poorer Black patients were at higher risk of death, mechanical ventilation, and ICU admission. This study is the first to identify median income as an independent predictor of IMV and ICU admission in COVID-19, even after adjusting for age, sex, and comorbidities. Analysis stratified by race revealed that male sex, obesity, and lower neighborhood median income were more significant predictors of poor outcomes for Black patients than for White patients. The disproportionate impact of neighborhood disadvantage on Black patients is likely due to the large income disparity between Black and White patients (neighborhood median income: $\$ 34,758$ vs. $\$ 63,317, p<0.001)$. We ground these income disparities on the structural inequities that drive the racial income and wealth gap in the USA. ${ }^{39-41}$

Our findings indicate that the racial disparities associated with the COVID-19 pandemic are not due to intrinsic characteristics of racial groups, but rather are produced by systemic inequities rooted in structural racism. These inequities affect income, educational attainment, food security, and healthcare. Therefore, social and legislative policies are critical in order to reduce socioeconomic disparities experienced by Black, 
Indigenous, and people of color (BIPOC) and can inform emerging initiatives such as the recently formed Michigan Coronavirus Task Force on Racial Disparities and Black Leadership Advisory Council. ${ }^{42,43}$ Solutions should be multi-level, synchronous, and coordinated. An intervention consisting of focused testing efforts with culturally competent strategies and messaging should target the most disadvantaged areas, which can be identified through the census. Upon hospital admission, appropriate screening for $\mathrm{SDOH}$ should be conducted to inform discharge planning, whereby patients from disadvantaged neighborhoods are provided additional resources for support. The healthcare system should also strive to better understand and eliminate institutional and clinician biases and address systemic racism in healthcare that hinders disease management and preventive care in underserved communities. ${ }^{44-46}$

Our study has limitations worth noting. Primarily, the study was retrospective in nature and collected data from one integrated healthcare system in southeast Michigan, limiting generalizability to other locations. Although this study examines the impact of race in COVID-19 outcomes, it focuses mainly on Black and White patients. Hispanic patients and nonEnglish speakers made up a small percentage of the cohort, limiting the conclusions that could be drawn for those subpopulations. There are large Middle Eastern, Latinx, and South Asian communities in metropolitan Detroit that were also not explicitly accounted for in the study. Further studies or larger cohorts could provide additional insight into the role of not only race but also ethnicity on COVID-19 outcomes. Another limitation is our use of census tract median income as a surrogate measure of neighborhood disadvantage. Though median income correlates with other socioeconomic measures on the census tract level, there may be high variability within a census tract that we did not account for. We were unable to obtain socioeconomic measures on the individual level and census tract socioeconomic measures may not accurately portray each individual's situation, which may change the variance in our effect estimates.

Our study examines one of the largest cohorts of hospitalized COVID-19 patients in an urban area with in-depth focus on the systemic inequities that impact social determinants of health. Detroit is home to one of the largest populations of Black Americans in the USA, and some of the nation's largest racial and health disparities, making it the ideal location to study and identify solutions to these deep-seated problems. The collection of social and environmental characteristics in our study sheds light on the large socioeconomic differences between Black and White patients with COVID-19. We identify neighborhood median income as a significant and independent predictor of severe disease, highlighting the need for multi-level approaches to reduce these disparities.

Supplementary Information The online version contains supplementary material available at https://doi.org/10.1007/s11606-02006527-1.
Acknowledgments The authors wish to acknowledge the additional contributions made by George Alangaden, MD; Indira Brar, MD; Anne Chen, MD; Erica Herc, MD; John E McKinnon, MD; Linoj Samuel, $P h D$; Mayur Ramesh, MD; Michael Reaume, MD; Robert Tibbetts, PhD; Geehan Suleyman, MD; Nicholas Yared, MD; and the Henry Ford COVID-19 Task Force.

Corresponding Author: Marcus Zervos, MD; Global Affairs Professor of Medicine, Assistant Dean Wayne State University School of Medicine, , MIDetroit, USA (e-mail: mzervos1@hfhs.org).

\section{Compliance with Ethical Standards}

Conflict of Interest The authors declare that they do not have a conflict of interest.

Open Access This article is licensed under a Creative Commons Attribution 4.0 International License, which permits use, sharing, adaptation, distribution and reproduction in any medium or format, as long as you give appropriate credit to the original author(s) and the source, provide a link to the Creative Commons licence, and indicate if changes were made. The images or other third party material in this article are included in the article's Creative Commons licence, unless indicated otherwise in a credit line to the material. If material is not included in the article's Creative Commons licence and your intended use is not permitted by statutory regulation or exceeds the permitted use, you will need to obtain permission directly from the copyright holder. To view a copy of this licence, visit http://creativecommons. org/licenses/by/4.0/.

\section{REFERENCES}

1. Centers for Disease Control and Prevention. CDC COVID Data Tracker; https://covid.cdc.gov/covid-data-tracker/\#cases_casesinlast7days.

2. Byrne P, McNamara B, Todd B, Tanner $\mathbf{K}$. Michigan coronavirus cases: Tracking the pandemic. 2020; https://www.freep.com/in-depth/news/ nation/coronavirus/2020/04/11/michigan-coronavirus-cases-trackingcovid-19-pandemic/5121186002/.

3. Whitmer G. Whitmer: Virus trains harsh spotlight on racial health disparities. 2020; https://www.freep.com/story/opinion/contributors/ 2020/04/12/whitmer-coronavirus-racial-health-disparities-michigan/ $5134653002 /$

4. The Covid Tracking Project. Racial Data Dashboard. 2020; https:// covidtracking.com/race/dashboard.

5. Guan WJ, Liang WH, Zhao Y, et al. Comorbidity and its impact on 1590 patients with COVID-19 in China: a nationwide analysis. Eur Respir J. 2020;55(5).

6. Yang $\mathbf{J}$, Zheng $\mathbf{Y}$, Gou $\mathbf{X}$, et al. Prevalence of comorbidities and its effects in patients infected with SARS-CoV-2: a systematic review and metaanalysis. Int J Infect Dis. 2020;94:91-95.

7. Price-Haywood EG, Burton J, Fort D, Seoane L. Hospitalization and Mortality among Black Patients and White Patients with Covid-19. N Engl J Med. 2020;382(26):2534-2543.

8. Suleyman G, Fadel RA, Malette KM, et al. Clinical Characteristics and Morbidity Associated With Coronavirus Disease 2019 in a Series of Patients in Metropolitan Detroit. JAMA Netw Open. 2020;3(6):e2012270.

9. Mikami T, Miyashita H, Yamada T, et al. Risk Factors for Mortality in Patients with COVID-19 in New York City. J Gen Intern Med. 2020.

10. Cummings MJ, Baldwin MR, Abrams D, et al. Epidemiology, clinical course, and outcomes of critically ill adults with COVID-19 in New York City: a prospective cohort study. Lancet. 2020;395(10239):1763-1770.

11. Adhikari K, Patten SB, Williamson T, et al. Does neighborhood socioeconomic status predict the risk of preterm birth? A communitybased Canadian cohort study. BMJ Open. 2019;9(2):e025341.

12. Galea S, Ahern J, Nandi A, Tracy M, Beard J, Vlahov D. Urban neighborhood poverty and the incidence of depression in a populationbased cohort study. Ann Epidemiol. 2007;17(3):171-179.

13. Brindle PM, McConnachie A, Upton MN, Hart CL, Davey Smith G, Watt GC. The accuracy of the Framingham risk-score in different socioeconomic groups: a prospective study. Br J Gen Pract. 2005;55(520):838-845.

14. McNicholas C, Poydock M. Who are essential workers? A comprehensive look at their wages, demographics, and unionization rates. 2020; 
https://www.epi.org/blog/who-are-essential-workers-a-comprehensivelook-at-their-wages-demographics-and-unionization-rates/.

15. United States Department of Agriculture Economic Research Service. Food Access Research Atlas. 2015; https://www.ers.usda.gov/dataproducts/food-access-research-atlas/.

16. United States Census Bureau. American Community Survey (ACS) 2018; https://www.census.gov/programs-surveys/acs.

17. Krieger N, Waterman P, Chen JT, Soobader MJ, Subramanian SV, Carson R. Zip code caveat: bias due to spatiotemporal mismatches between zip codes and US census-defined geographic areas-the Public Health Disparities Geocoding Project. Am J Public Health. 2002;92(7):1100-1102.

18. Schootman M, Jeffe DB, Lian M, et al. Area-level poverty is associated with greater risk of ambulatory-care-sensitive hospitalizations in older breast cancer survivors. J Am Geriatr Soc. 2008;56(12):2180-2187.

19. Freeman VL, Ricardo AC, Campbell RT, Barrett RE, Warnecke RB. Association of census tract-level socioeconomic status with disparities in prostate cancer-specific survival. Cancer Epidemiol Biomarkers Prev. 2011;20(10):2150-2159.

20. Census Reporter. https://censusreporter.org/.

21. Laurencin CT, McClinton A. The COVID-19 Pandemic: a Call to Action to Identify and Address Racial and Ethnic Disparities. J Racial Ethn Health Disparities. 2020;7(3):398-402.

22. The COVID Racial Data Tracker. 2020; https://covidtracking.com/race.

23. Lewnard JA, Lo NC. Scientific and ethical basis for social-distancing interventions against COVID-19. Lancet Infect Dis. 2020;20(6):631-633.

24. Dorn AV, Cooney RE, Sabin ML. COVID-19 exacerbating inequalities in the US. Lancet. 2020;395(10232): 1243-1244.

25. Yancy CW. COVID-19 and African Americans. JAMA. 2020.

26. Anderson E, Hall C, Tanner K. 3 Michigan nursing homes report more than 100 coronavirus cases. 2020; https://www.freep.com/story/news/ local/michigan/2020/05/29/nursing-homes-michigan-coronavirus / $5282914002 /$.

27. Emeruwa UN, Ona S, Shaman JL, et al. Associations Between Built Environment, Neighborhood Socioeconomic Status, and SARS-CoV-2 Infection Among Pregnant Women in New York City. JAMA. 2020.

28. Tamara A, Tahapary DL. Obesity as a predictor for a poor prognosis of COVID-19: A systematic review. Diabetes Metab Syndr. 2020;14(4):655659.

29. Simonnet A, Chetboun M, Poissy J, et al. High Prevalence of Obesity in Severe Acute Respiratory Syndrome Coronavirus-2 (SARS-CoV-2) Requiring Invasive Mechanical Ventilation. Obesity (Silver Spring). 2020;28(7):1195-1199.

30. Gooding HC, Walls CE, Richmond TK. Food insecurity and increased BMI in young adult women. Obesity (Silver Spring). 2012;20(9):18961901.

31. Larson NI, Story MT, Nelson MC. Neighborhood environments: disparities in access to healthy foods in the U.S. Am J Prev Med. 2009;36(1):74 81.
32. Spence JC, Cutumisu N, Edwards J, Raine KD, Smoyer-Tomic K. Relation between local food environments and obesity among adults. BMC Public Health. 2009;9:192.

33. Alang S, McAlpine D, McCreedy E, Hardeman R. Police Brutality and Black Health: Setting the Agenda for Public Health Scholars. Am J Public Health. 2017;107(5):662-665.

34. DeGue S, Fowler KA, Calkins C. Deaths Due to Use of Lethal Force by Law Enforcement: Findings From the National Violent Death Reporting System, 17 U.S. States, 2009-2012. Am J Prev Med. 2016;51(5 Suppl 3):S173-S187.

35. Gilbert KL, Ray R, Siddiqi A, et al. Visible and Invisible Trends in Black Men's Health: Pitfalls and Promises for Addressing Racial, Ethnic, and Gender Inequities in Health. Annu Rev Public Health. 2016;37:295-311.

36. Laurencin CT, Murray M. An American Crisis: the Lack of Black Men in Medicine. J Racial Ethn Health Disparities. 2017;4(3):317-321.

37. Massoglia M, Firebaugh G, Warner C. Racial Variation in the Effect of Incarceration on Neighborhood Attainment. Am Sociol Rev. 2013;78(1).

38. Taylor RJ, Miller R, Mouzon D, Keith VM, Chatters LM. Everyday Discrimination among African American Men: The Impact of Criminal Justice Contact. Race Justice. 2018;8(2):154-177.

39. Williams DR, Mohammed SA, Leavell J, Collins C. Race, socioeconomic status, and health: complexities, ongoing challenges, and research opportunities. Ann N Y Acad Sci. 2010;1186:69-101.

40. Krivo LJ, Kaufman RL. Housing and wealth inequality: racial-ethnic differences in home equity in the United States. Demography. 2004;41(3):585-605.

41. Nuru-Jeter AM, LaVeist TA. Racial segregation, income inequality, and mortality in US metropolitan areas. J Urban Health. 2011;88(2):270-282.

42. Michigan Coronavirus Task Force on Racial Disparities. Executive Order 2020-55 (COVID-19). 2020; https://www.michigan.gov/whitmer/ 0,9309,7-387-90499_90705-526476\%2D\%2D,00.html.

43. Whitmer. TOoGG. Governor Whitmer Signs Executive Directive Recognizing and Addressing Racism as a Public Health Crisis, Creates the Black Leadership Advisory Council. 2020; https://www.michigan.gov/ whitmer/0,9309,7-387-90499-535762\%2D\%2D,00.html

44. Hall WJ, Chapman Mv, Lee KM, et al. Implicit Racial/Ethnic Bias Among Health Care Professionals and Its Influence on Health Care Outcomes: A Systematic Review. Am J Public Health. 2015;105(12):e6076.

45. Shen MJ, Peterson EB, Costas-Muniz R, et al. The Effects of Race and Racial Concordance on Patient-Physician Communication: A Systematic Review of the Literature. J Racial Ethn Health Disparities. 2018;5(1):117140.

46. Obermeyer Z, Powers B, Vogeli C, Mullainathan S. Dissecting racia bias in an algorithm used to manage the health of populations. Science. 2019;366(6464):447-453.

Publisher's Note Springer Nature remains neutral with regard to jurisdictional claims in published maps and institutional affiliations. 